Research Article

\title{
New Cubic Trigonometric Bezier-Like Functions with Shape Parameter: Curvature and Its Spiral Segment
}

\author{
Abdul Majeed ${ }^{1},{ }^{1}$ Muhammad Abbas ${ }^{D},{ }^{2}$ Amna Abdul Sittar, ${ }^{1}$ Mohsin Kamran, ${ }^{1}$ \\ Saba Tahseen, ${ }^{1}$ and Homan Emadifar $\mathbb{D}^{3}$ \\ ${ }^{1}$ Department of Mathematics, Division of Science and Technology, University of Education, Lahore, Pakistan \\ ${ }^{2}$ Department of Mathematics, University of Sargodha, Sargodha 40100, Pakistan \\ ${ }^{3}$ Department of Mathematics, Islamic Azad University, Hamedan Branch, Hamedan, Iran \\ Correspondence should be addressed to Muhammad Abbas; muhammad.abbas@uos.edu.pk and Homan Emadifar; \\ homan_emadi@yahoo.com
}

Received 4 July 2021; Revised 25 August 2021; Accepted 2 September 2021; Published 13 September 2021

Academic Editor: Ali Ahmad

Copyright $\odot 2021$ Abdul Majeed et al. This is an open access article distributed under the Creative Commons Attribution License, which permits unrestricted use, distribution, and reproduction in any medium, provided the original work is properly cited.

\begin{abstract}
This work presents the new cubic trigonometric Bézier-type functions with shape parameter. Basis functions and the curve satisfy all properties of classical Bézier curve-like partition of unity, symmetric property, linear independent, geometric invariance, and convex hull property and have been proved. The $C^{3}$ and $G^{3}$ continuity conditions between two curve segments have also been achieved. To check the applicability of proposed functions, different types of open and closed curves have been constructed. The effect of shape parameter and control points has been observed. It is observed that, by decreasing the value of shape parameter, the curve moves toward the control polygon and vice versa. The CT-Bézier curve is closer to the cubic Bézier curve for a fixed value of shape parameter. The proposed CTBézier curve can be used to represent ellipse. Using proposed basis functions, we have constructed the spiral segment which is very useful to construct fair curves and desirable to design trajectories of mobile robots, highway, and railway routes' designing.
\end{abstract}

\section{Introduction}

Spline curves have been considered a major tool for the geometric modelling in computer aided geometric design. In recent past, trigonometric Bézier-like functions and curves have also attained the attention in computer aided geometric design, computer aided design, and bio-modelling $[1,2]$. The concept of trigonometric B-spline (TBS) was introduced by [3], and the scheme of trigonometric B-spline with recurrence relation of the arbitrary order has been presented in [4]. A technique based on cubic Bézier curves (CBC) with the association of a shape parameter is proposed by [5]. This technique is useful for the construction of planer curves and the shape parameter is used to control the curve. Han et al. [6] proposed the trigonometric cubic Bézier curves with two shape parameters. Spiral segments are considered useful to construct fair curves and desirable to design trajectories of mobile robots, highway, and railway routes' designing. The scheme proposed in [7] is suitable for the " $S$ " shaped curves.
Spiral and transition curves have been constructed using CTB with appropriate conditions in [8]. And, this work has been extended to cubic Bézier curve and Bézier-like curves with exponential functions in [9].

A technique, based on quadratic trigonometric Bézier (QTB) basis functions using one shape parameter, has been introduced in [10]. Generalized trigonometric Bézier curves with one shape parameter is introduced in [11]. For corner cutting algorithm, Bosner and Rogina [12] have proposed the cycloidal splines. Wen and Wang [13] have proposed the uniform trigonometric $B$-spline of order $n^{\text {th }}$ with shape parameters. These basis functions are very suitable for designing the circular and elliptic type objects. Class of nonuniform B-spline basis functions with local shape parameter is presented in [14]. Using these nonuniform basis functions, one can attain the $C^{2}$ continuity for single knot and $C^{3}$ and $C^{5}$ continuity can be attained for unique shape parameters. Han [15] demonstrated the trigonometric cubic B-spline with exponential shape parameter. Cubic B-spline 
basis functions on uniform knot with one shape parameter are proposed in [16]. Chouby and Ojha [17] proposed the trigonometric spline curve. In this scheme, the shape parameter is a variable which is helpful in adjusting and controlling the curve and surface locally. Denominator trigonometric DT-B-spline basis function $i$ proposed in [18] is similar to trigonometric B-spline functions. These functions have denominator shape parameter. Troll [19] introduced the trigonometric cubic Bézier curve with constrained and two shape parameters. Trigonometric B-spline basis functions of degree 2 and the quadratic NUAT-B-spline curve of many shape parameters are proposed in $[20,21]$. Cubic trigonometric B-spline curve has been proposed in $[22,23]$. Xie and Li [24] proposed the cubic trigonometric B-spline basis and curve with real shape parameter called alpha-B-spline curve. Hang et al. [25] proposed the cubic B-spline curve with shape parameter and mainly focus on the quasi-uniform B-spline curve. The authors used the proposed the curve for generating the fractal curves. Hu et al. [26] proposed the generalized developable surface shape parameters. The generalized developable $H$-Bézier surfaces are designed by using control planes with generalized $\mathrm{H}$-Bézier basis functions, and their shapes can be adjusted by altering the values of shape parameters. Kovcs and Vrady [27] introduced P-Bézier and P-B-spline curve. Cubic B-spline collocation method has been used in [28] for the numerical solution of time fractional advection diffusion equation. Crank-Nicolson with cubic B-spline has been used in [29] for the solution parabolic partial differential equation.

In this work, new trigonometric Bézier basis functions with a shape parameter are constructed. The proposed bases are more efficient as the degree of proposed bases is two but it works with four control points. We have also constructed the spiral segment using the proposed bases which is not common in literature, using trigonometric functions. The proposed basis functions and the curve satisfy all basic properties such as partition of unity, linear independency, symmetric property, convex hull property, and geometric invariance. Different curve segments are constructed using proposed basis functions. The $C^{3}$ and $G^{3}$ continuity conditions are also discussed. The shape of the curve can be rearranged by varying values of the shape parameter. The proposed CT-Bézier curve behaves like cubic Bézier curve for a specific value of the shape parameter. By decreasing the value of the shape parameter, the curve gets closer to the control polygon. The ellipse can be represented exactly using proposed cubic trigonometric Bézier curve. To illustrate the application of proposed cubic Bézier curves, different open and closed curves are designed; the constructed curves are very flexible and easy to handle.

The present work is organized as follows. In Section 2, new proposed cubic trigonometric bases functions and their properties are described. Cubic trigonometric Bézier curves, their properties, effect of shape parameter, and parametric and geometric continuity are part of Section 3. In Section 4, application of the proposed curve is discussed. In Sections 5 and 6, representation of ellipse and approximation of cubic trigonometric Bézier curve to the ordinary cubic Bézier curve is presented. In Section 7, curvature and spiral curves are discussed, and Section 8 is all about conclusions.

\section{Cubic Trigonometric Bézier Functions}

For a shape parameter $m \varepsilon[0,1]$, the proposed trigonometric Bézier-like functions $b_{i}(u), i=0, \ldots, 3$, are defined as

$$
\begin{aligned}
& b_{0}(u)=\left(1-\sin \frac{\pi}{2} u\right)\left[\left(1-\sin \frac{\pi}{2} u\right)+m \sin \frac{\pi}{2} u\right], \\
& b_{1}(u)=\sin \frac{\pi}{2} u\left(1-\sin \frac{\pi}{2} u\right)(2-m), \\
& b_{2}(u)=\cos \frac{\pi}{2} u\left(1-\cos \frac{\pi}{2} u\right)(2-m), \\
& b_{3}(u)=\left(1-\cos \frac{\pi}{2} u\right)\left[\left(1-\cos \frac{\pi}{2} u\right)+m \cos \frac{\pi}{2} u\right] .
\end{aligned}
$$

The graphical behavior of proposed basis functions defined in equation (1) can be observed in Figure 1. The effect of shape parameter $m$ can also be observed in this figure.

\subsection{Properties of the Basis Functions}

Theorem 1. Proposed trigonometric basis functions defined in equation (1) satisfy the following properties:

Positivity: all trigonometric functions are positive, i.e., $b_{j}(u) \geq 0$, for $j=0, \ldots, 3$

Partition of unity: sum of all trigonometric functions is one, mathematically, $\sum_{i=0}^{3} b_{i}=1$

Symmetry: proposed functions are symmetric means; $b_{0}$ becomes $b_{3}$ and vice versa by replacing $u$ by $u-1$; mathematically, $b_{i}(u ; m)=b_{3-i}(1-u ; m), \quad$ for $i=0, \ldots, 3$

Linearly independent: the basic functions are linearly independent, as they cannot be written as a linear combination of each other for any nonzero constant $p_{0}, \ldots p_{3}$

Proof. (a) For $u \in[0,1]$ and $m \in[0,1]$, then

$$
\begin{aligned}
& 0 \leq(1-\sin (\pi / 2) u)^{2} \leq 1 \\
& 0 \leq(1-\sin (\pi / 2) u)(m \sin (\pi / 2) u) \leq 1 \\
& 0 \leq(1-\cos (\pi / 2) u)^{2} \leq 1 \\
& 0 \leq(1-\cos (\pi / 2) u))(m \cos (\pi / 2) u) \leq 1
\end{aligned}
$$

It is observed that $b_{i} \geq 0, i=0,1,2,3$.

(b) $\quad \sum_{i=0}^{3} b_{i}(u)=(1-\sin (\pi / 2) u)[((1-\sin (\pi / 2) u)+m$ $\sin (\pi / 2) u)]+((\sin (\pi / 2) u)(1-\sin (\pi / 2) u)(2-m)+(\cos$ $(\pi / 2) u)(1-\cos (\pi / 2) u))(2-m)+(1-\cos (\pi / 2) u)[((1$ $-\cos (\pi / 2) u)+m \cos (\pi / 2) u)]=1$.

The remaining cases follow obviously. 


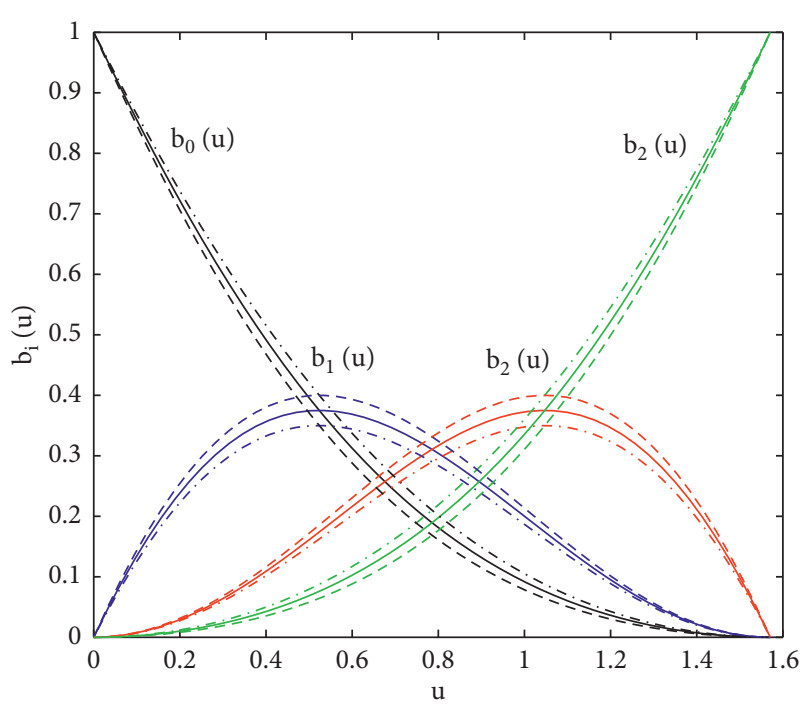

Figure 1: Cubic trigonometric functions with different shape parameters.

\section{Trigonometric Cubic Bézier Curve}

For given control points $p_{i}(i=0,1,2,3)$ in $R^{2}$ or $R^{3}$, the cubic trigonometric Bézier curve with a shape parameter $m$ is defined as

$$
r(u)=\sum_{i=0}^{3} b_{i} p_{i}, \quad u \in[0,1], m \in[0,1]
$$

\subsection{Properties of CT-Bézier Curve}

Theorem 2. The CT-Bézier curve defined in equation (2) satisfies the following properties:

\section{Endpoint interpolation:}

Cubic trigonometric curve always passes through the first and last control point:

$$
\begin{aligned}
& r(0)=p_{0}, \\
& r(1)=p_{3} .
\end{aligned}
$$

\section{Geometric invariance:}

The shape of a cubic trigonometric Bézier curve is independent of the choice of coordinates; i.e., equation (2) satisfies the following two equations:

$$
\begin{aligned}
& r\left(u ; m ; p_{0}+q, p_{1}+q, p_{2}+q, p_{3}+q\right)=r\left(u ; m ; p_{0}, p_{1}, p_{2}, p_{3}\right)+q, \\
& r\left(u ; m ; p_{0}+T, p_{1}+T, p_{2}+T, p_{3}+T\right)=r\left(u ; m ; p_{0}, p_{1}, p_{2}, p_{3}\right)+T,
\end{aligned} \quad t \in[0,1], m \in[0,1],
$$

where $q$ is arbitrary vector in $R^{2}$ or $R^{3}$ and $T$ is an arbitrary $d \times d$ matrix, $d=2$ or 3 .

Convex hull property:

The cubic curve always lies within the convex hull of control polygon.

Coordinate system independence:

The proposed curve is independent of the coordinate system means by changing the coordinated curve remains unchanged.

3.2. Continuity Conditions between Two Curve Segments. In this section, we will derive the different parametric and geometric continuity conditions between two curve segments.

3.2.1. Parametric Continuity. (i) $C^{0}$ Continuity $\left(p_{3}=q_{0}\right)$. It is obvious, which means $C_{0}$ continuity holds.

(ii) $C^{1}$ Continuity.

$$
\begin{aligned}
& r^{\prime}(1)=\frac{\pi}{2}(2-m)\left(p_{3}-p_{2}\right), \\
& r_{1}^{\prime}(1)=\frac{\pi}{2}(2-m)\left(q_{1}-q_{0}\right),
\end{aligned}
$$

as $p_{3}=q_{0}$ and $r^{\prime}(1)=r_{1}^{\prime}(0)$, so $C_{1}$ continuity holds.

(iii) $C^{2}$ Continuity.

$$
\begin{aligned}
& r^{\prime \prime}(1)=\left(\frac{\pi}{2}\right)^{2}\left[m p_{0}+(2-m) p_{1}-2(2-m) p_{2}+(2-2 m) p_{3}\right] \\
& r_{1}^{\prime \prime}(1)=\left(\frac{\pi}{2}\right)^{2}\left[(2-2 m) q_{0}+2(2-m) q_{1}+(2-m) q_{2}+m q_{3}\right]
\end{aligned}
$$

as $p_{3}=q_{0}, r^{\prime}(1)=r_{1}^{\prime}(0)$, and $r^{\prime \prime}(1)=r_{1}^{\prime \prime}(0)$, so $C_{2}$ continuity holds.

(iv) $C^{3}$ Continuity.

$r^{\prime \prime \prime}(1)=(\pi / 2)^{3}(2-m)\left(p_{2}-p_{3}\right)$ and $r_{1}^{\prime \prime}(0)=(\pi / 2)^{3}(2-$ $m)\left(p_{0}-p_{1}\right) \quad$ as $\quad r^{\prime}(1)=r_{1}^{\prime}(0), \quad r^{\prime \prime}(1)=r_{1}^{\prime \prime}(0), \quad$ and $r^{\prime \prime \prime}(1)=r_{1}^{\prime \prime}(0)$, so $C_{3}$ continuity holds.

3.2.2. Geometric Continuity. (i) $G^{0}$ Continuity $\left(p_{3}=q_{0}\right)$. It is obvious, which means $G_{0}$ continuity holds.

(ii) $G^{1}$ Continuity.

$$
\begin{aligned}
r^{\prime}(1) & =\frac{\pi}{2}(2-m)\left(p_{3}-p_{2}\right), \\
r_{1}^{\prime}(1) & =\frac{\pi}{2}(2-m)\left(q_{1}-q_{0}\right), \\
\frac{\pi}{2}(2-m)\left(q_{1}-q_{0}\right) & =\lambda\left(\frac{\pi}{2}(2-m)\left(p_{3}-p_{2}\right)\right), \text { i.e., } \\
r_{1}^{\prime}(0) & =\lambda r^{\prime}(1) .
\end{aligned}
$$

All conditions are satisfied. So, $G_{1}$ continuity holds. (iii) $G^{2}$ Continuity. 


$$
\begin{aligned}
& r^{\prime \prime}(1)=\left(\frac{\pi}{2}\right)^{2}\left[m p_{0}+(2-m) p_{1}-2(2-m) p_{2}+(2-2 m) p_{3}\right], \quad p_{3}=q_{0}, \text { and } r_{1}^{\prime}(0)=\lambda r^{\prime}(1) ; \text { also, } \\
& r_{1}^{\prime \prime}(1)=\left(\frac{\pi}{2}\right)^{2}\left[(2-2 m) q_{0}+2(2-m) q_{1}+(2-m) q_{2}+m q_{3}\right],
\end{aligned}
$$

$$
\begin{aligned}
\left(\frac{\pi}{2}\right)^{2}\left[(2-2 m) q_{0}+2(2-m) q_{1}+(2-m) q_{2}+m q_{3}\right]= & \lambda^{2}\left(\frac{\pi}{2}\right)^{2}\left[m p_{0}+(2-m) p_{1}-2(2-m) p_{2}+(2-2 m) p_{3}\right] \\
& +\gamma \frac{\pi}{2}(2-m)\left(p_{3}-p_{2}\right)
\end{aligned}
$$

i.e., $r_{1}^{\prime \prime}(0)=\lambda^{2} r^{\prime \prime}(1)+\gamma r^{\prime}(1)$. So, $G_{2}$ continuity holds.

$$
\begin{aligned}
\left(\frac{\pi}{2}\right)^{3}(2-m)\left(p_{0}-p_{1}\right)= & \lambda^{3}\left(\frac{\pi}{2}\right)^{3}(2-m)\left(p_{2}-p_{3}\right)+\gamma^{2}\left(\frac{\pi}{2}\right)^{2}\left[m p_{0}+(2-m) p_{1}-2(2-m) p_{2}+(2-2 m) p_{3}\right] \\
& +\beta\left(\frac{\pi}{2}\right)(2-m)\left(p_{3}-p_{2}\right), \text { i.e., } \\
r_{1}^{\prime \prime \prime}(0)= & \lambda^{3} r^{\prime \prime \prime}(1)+\gamma^{2} r^{\prime \prime}(1)+\beta r^{\prime}(1) .
\end{aligned}
$$

(iv) $G^{3}$ Continuity. $r^{\prime \prime \prime}(1)=(\pi / 2)^{3}(2-m)\left(p_{2}-p_{3}\right)$, and $r_{1}^{\prime \prime}(0)=(\pi / 2)^{3}(2-m)\left(p_{0}-p_{1}\right)$ as $r^{\prime}(1)=r_{1}^{\prime}(0) p_{3}=q_{0}$, and $r_{1}^{\prime \prime}(0)=\lambda^{2} r^{\prime \prime}(1)+\gamma r^{\prime}(1)$; also,
All conditions are satisfied. So, $G_{3}$ continuity holds.

\section{Application of Proposed Curves}

In this section, different open and closed curves have been constructed using proposed functions. The effect of shape parameter $m$ and control points will also be observed in detail as in Figure 2; the open curve has been constructed using different values of $m$ as $m=0.1$ (green dashed dotted), $m=0.3$ (blue dotted), and $m=0.5$ (red solid), $m=0.7$ (black dotted), and $m=0.9$ (magenta dash dotted).

The effect of control point can be observed in Figures 3 and 4. In Figure 3, we constructed the two segment curve using seven control points. The effect of first and last control point is observed in this figure. The curve follows the direction of control point as the control point moves toward outside curve move in the same direction, as shown in Figure 3(b). Similarly, in Figure 3(d), the curve moves toward inside. Figure 4 represents the effect of second and second-last control point.

Another way to control the curve is the shape parameter. In this case, there is no need to change the control points, as shown in Figure 5. In this figure, the four-segment curve has been constructed using different values of $m$ such as $m=0.1$ (green dashed), $m=0.3$ (black dash dotted), $m=0.5$ (red solid), $m=0.7$ (blue dash dotted), and $m=0.9$ (magenta dashed). For $m=0$, the curve becomes a straight line.

In Figure 6, different values of $m$ are $m=0.1$ (magenta dotted), $m=0.3$ (black dash dotted), $m=0.5$ (red solid), $m=0.7$ (blue dash dotted), and $m=1$ (red dotted). The curves become the straight line when $m=0$. It is observed that, by increasing the value of $m$, the curve moves toward the control polygon, and by decreasing, it moves away from the control polygon.

To check the applicability of the proposed scheme, different closed curves using different shape parameters have also been designed in this paper, as shown in Figures 7 and 8 . In Figure 7, $m=0.1$ (magenta dashed), $m=0.3$ (red dash dotted), $m=0.5$ (black solid), $m=0.7$ (blue dashed), and $m=0.8$ (magenta dash dotted), and in Figure $8, m=0.1$ (green dashed), $m=0.3$ (black dash dotted), $m=0.5$ (red solid), $m=0.7$ (blue dash dotted), and $m=0.9$ (magenta dashed) have been used for designing.

\section{Representation of Ellipse}

Theorem 3. Let $p_{i}$ be control points for ellipse with semiaxes " $k$ " and " $n$;" for suitable coordinates, coordinates of ellipse can be written in the following form:

$$
\begin{aligned}
& p_{0}=\left(\begin{array}{c}
2 k \\
0
\end{array}\right), \\
& p_{1}=\left(\begin{array}{c}
2 k \\
n
\end{array}\right), \\
& p_{2}=\left(\begin{array}{c}
k \\
2 n
\end{array}\right), \\
& p_{3}=\left(\begin{array}{c}
0 \\
2 n
\end{array}\right) .
\end{aligned}
$$




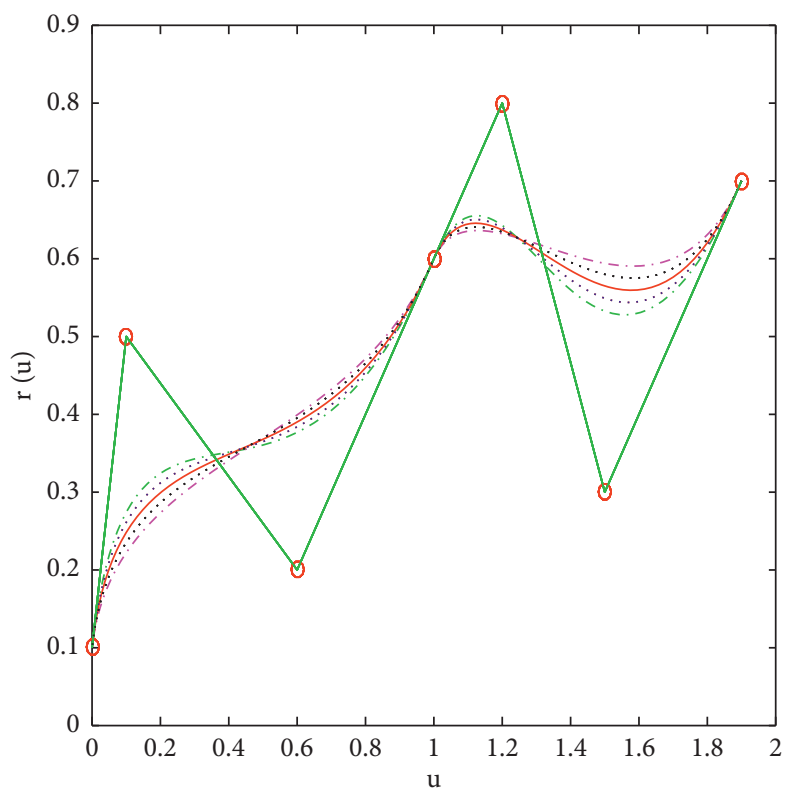

Figure 2: Open curve using CT-Bézier basis functions.

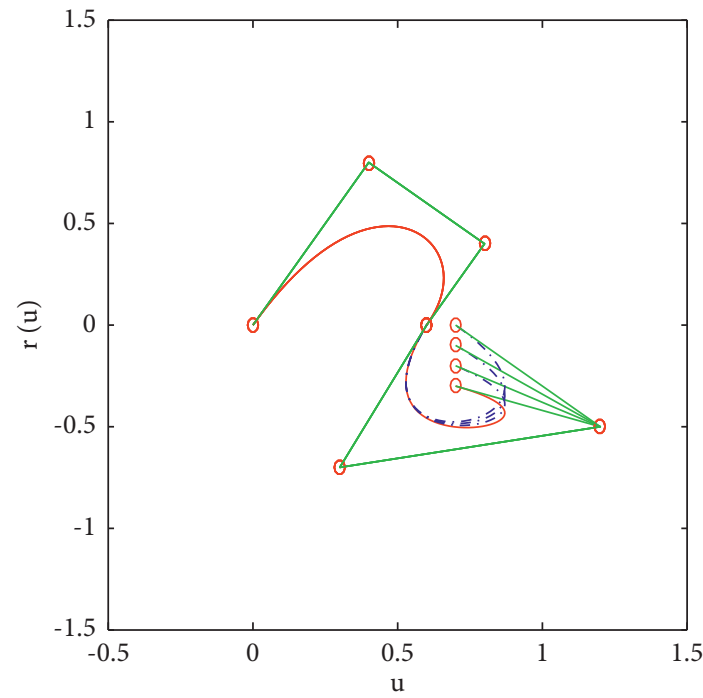

(a)

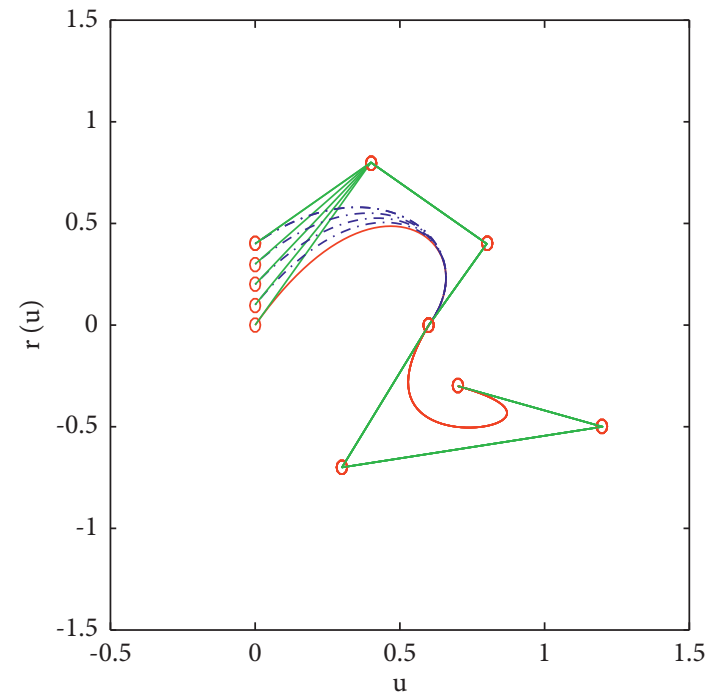

(b)

Figure 3: Continued. 


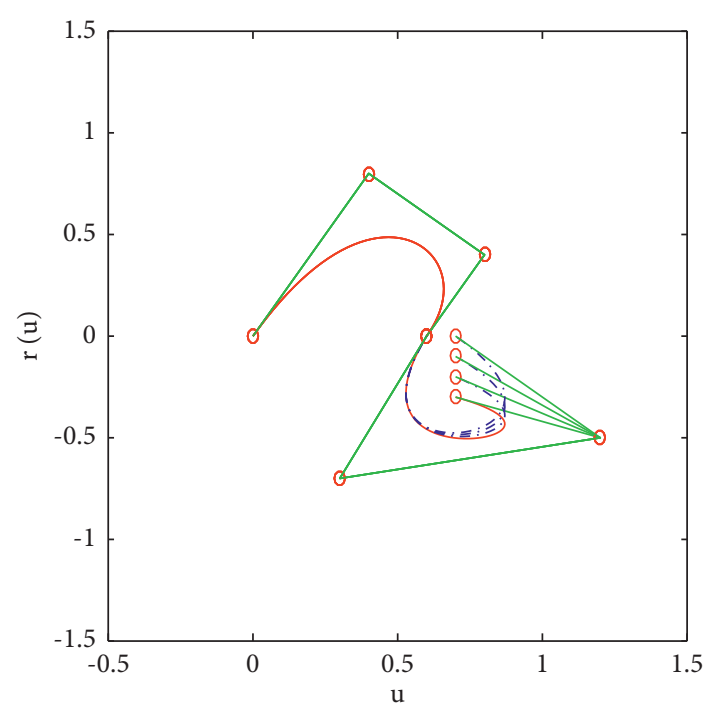

(c)

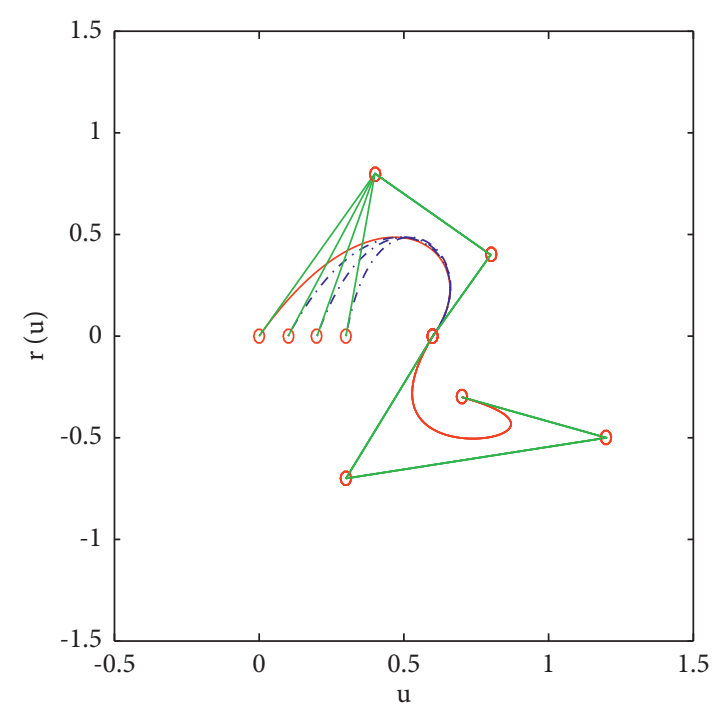

(d)

FIgURE 3: Effect of control points on open curve (a-d).

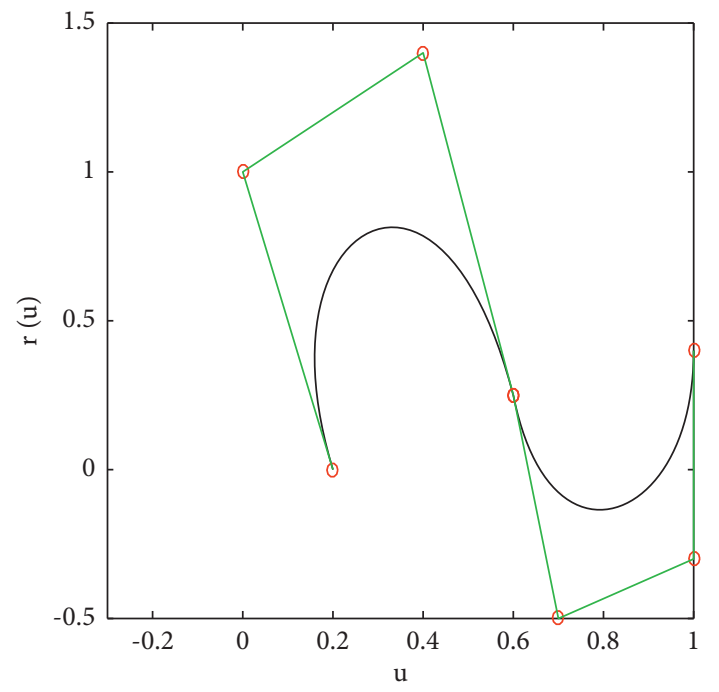

(a)

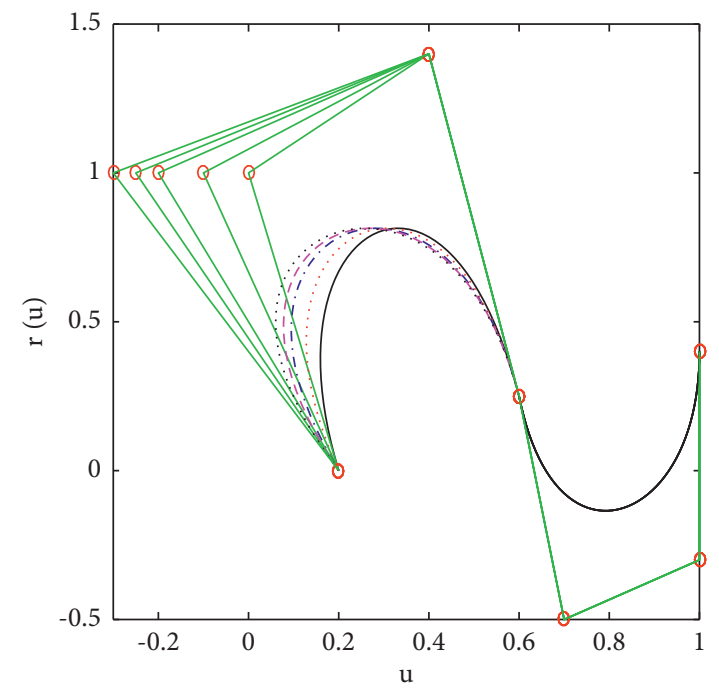

(b)

FIgURE 4: Continued. 


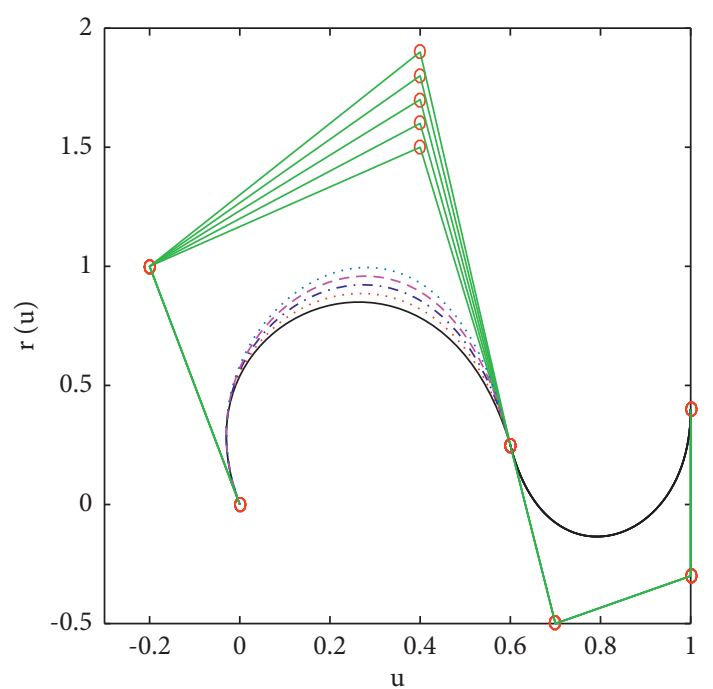

(c)

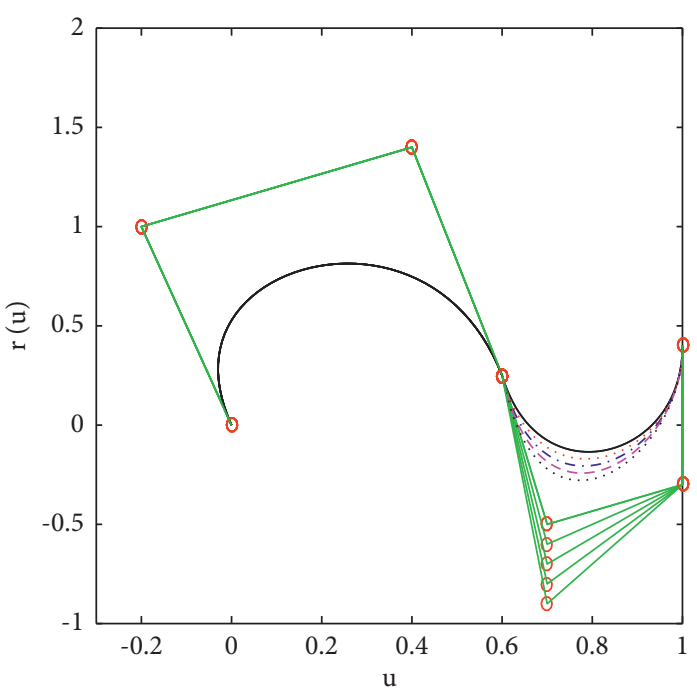

(d)

Figure 4: Effect of control points (a-d).

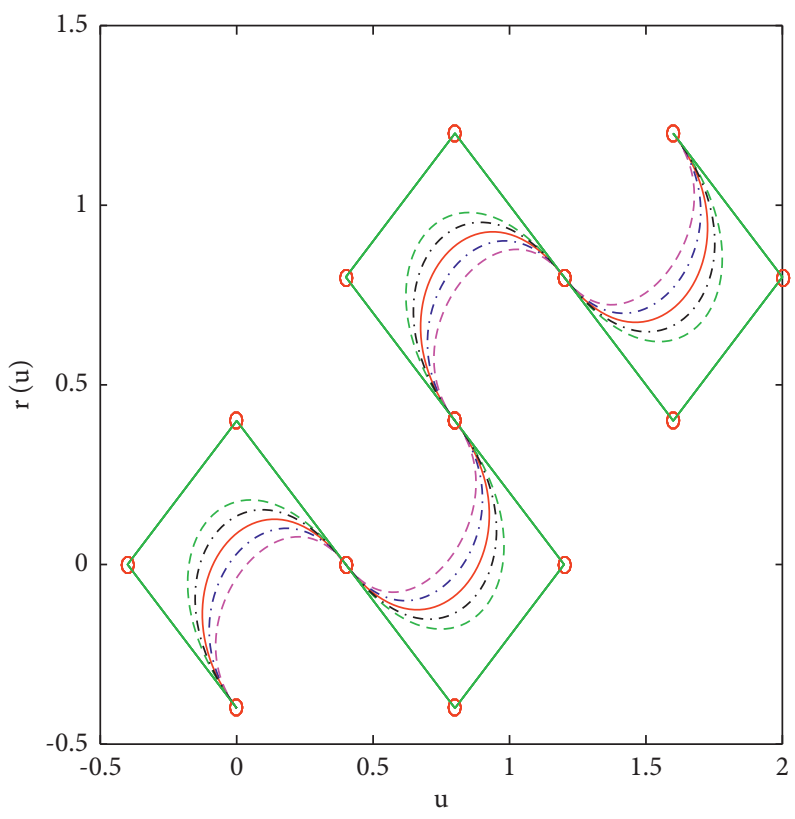

FIGURE 5: The effect of the shape parameter.

Then, the corresponding CT-Bézier curve with shape parameter $m=0$ and local domain $u \in[0,1]$ represents an arc of a'n ellipse with

$$
\begin{aligned}
& r_{x}(u)=2 k \cos \frac{\pi}{2} u, \\
& r_{y}(u)=2 n \sin \frac{\pi}{2} u .
\end{aligned}
$$

Proof. If we put the given points in equation (2), coordinates of CT-Bézier curve becomes

$$
\left\{\begin{array}{l}
x(u)=2 k \cos \frac{\pi}{2} u, \\
y(u)=2 k \sin \frac{\pi}{2} u .
\end{array}\right.
$$

This gives the intrinsic equation:

$$
\left(\frac{x}{2 k}\right)^{2}+\left(\frac{y}{2 n}\right)^{2}=1
$$

It is equation of ellipse, and Figure 9 represents the graphical behavior of ellipse. 


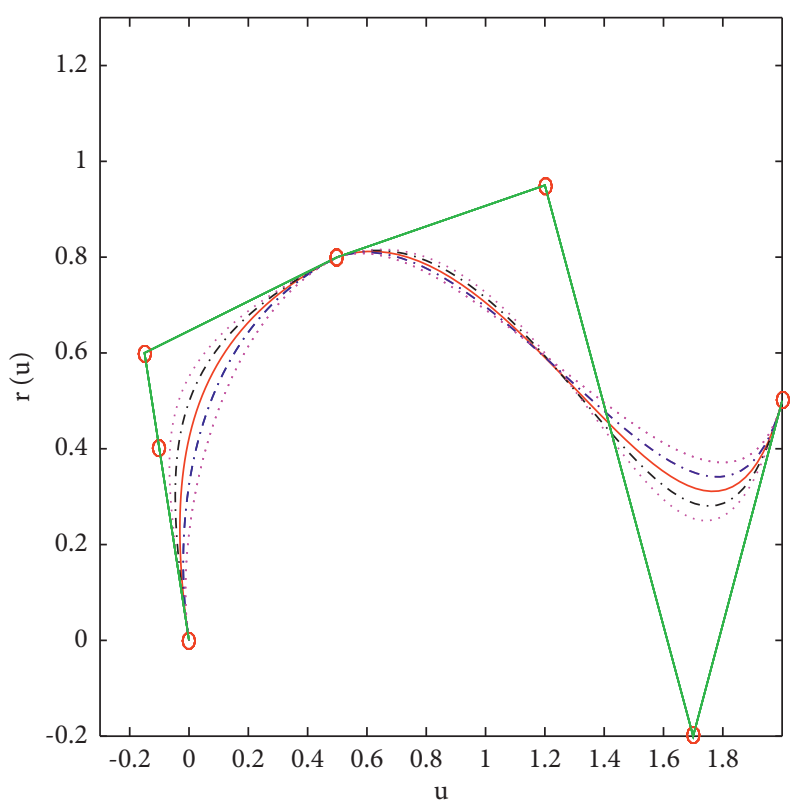

FIgURE 6: The effect of different values of $m$.

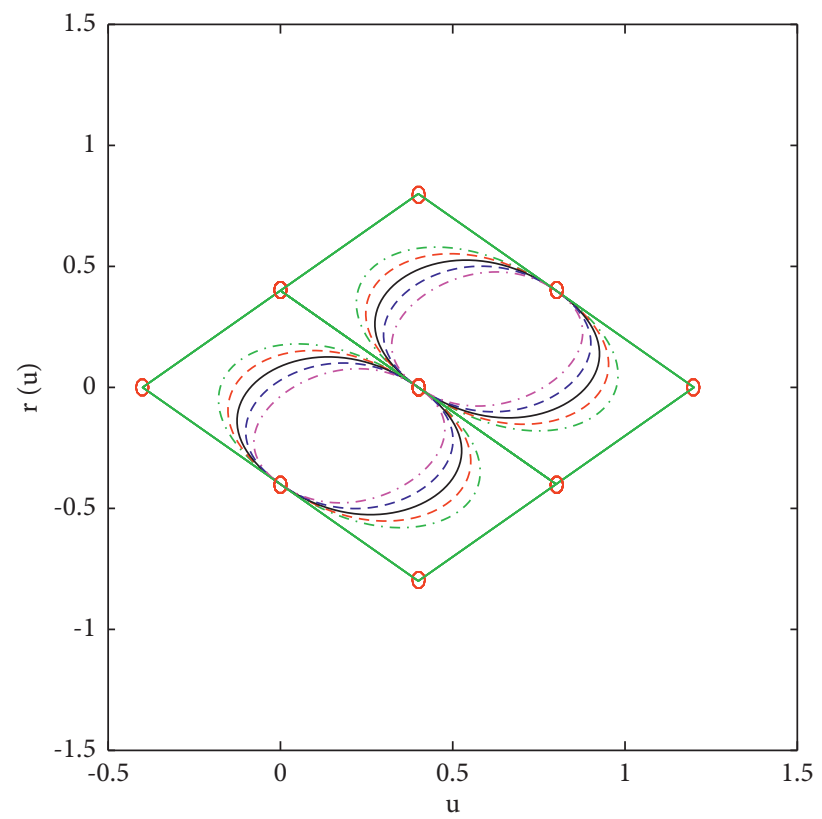

Figure 7: Closed curve using CT-Bézier.

\section{Approximability}

For curve construction, the control polygon plays a vital role. In this section, we will develop the relation between the classic and trigonometric Bézier curve corresponding to their control polygons by adjusting the control point and shape parameter.

Theorem 4. For noncollinear control points $p_{0}, p_{1}, p_{2}$, and $p_{3}$, the relation between classical and trigonometric Bézier curve $B(u)=\sum_{i=0}^{3} p_{i}\left(\begin{array}{l}3 \\ 1\end{array}\right)(1-u)^{3-i} u^{i}, u \in[0,1]$, with control points $p_{i}(i=0, \ldots, 3)$ are as follows:

$$
\left\{\begin{array}{l}
r(0)=B(0) \\
r(1)=B(1)
\end{array}\right.
$$

$$
r\left(\frac{1}{2}\right)-P^{\prime}=4(\sqrt{ } 2-1)(\sqrt{ } 2-1+m)\left(B\left(\frac{1}{2}\right)-P^{\prime}\right),
$$




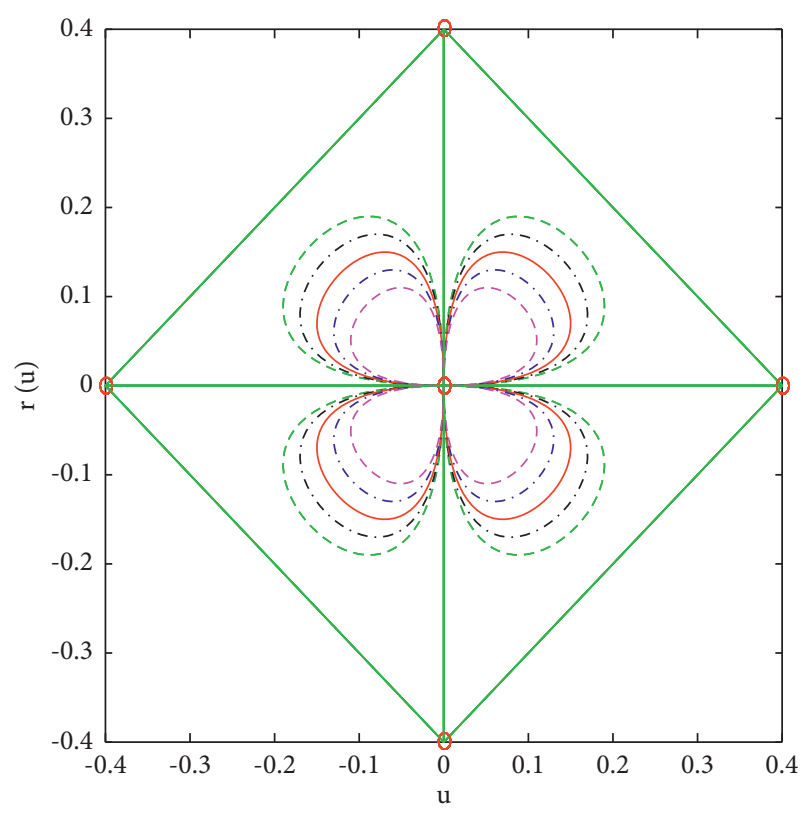

Figure 8: Flower designing using CT-Bézier basis functions.

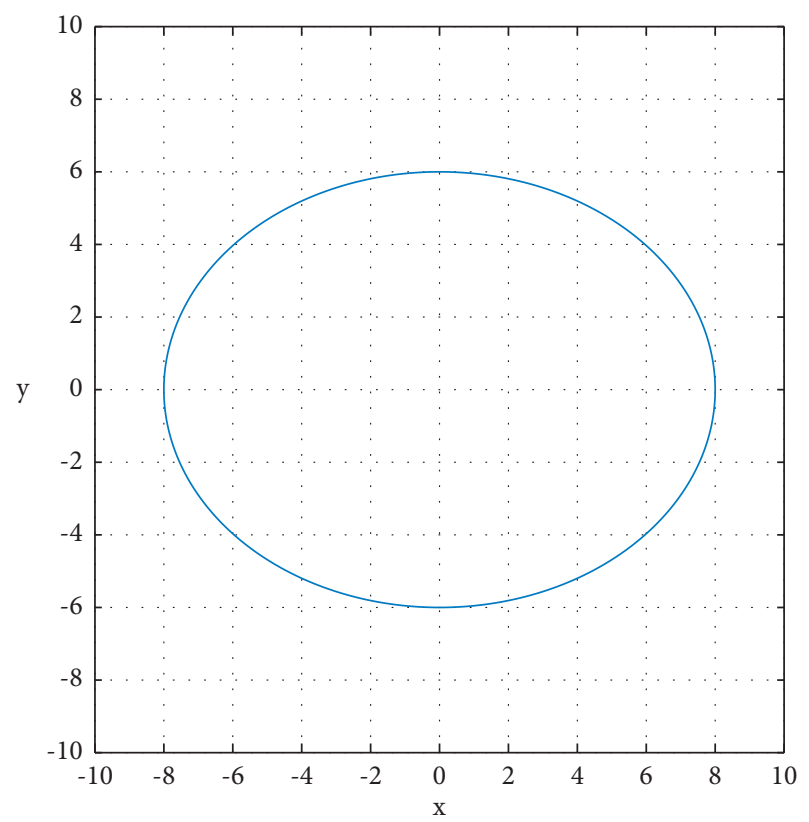

Figure 9: Representation of ellipse using the CT-Bézier curve.

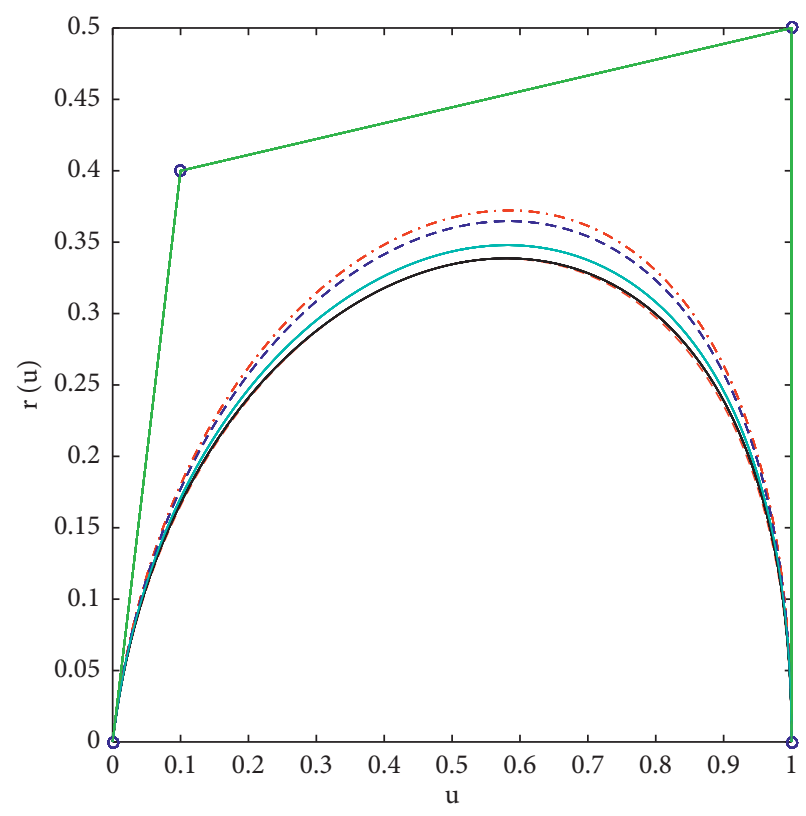

Figure 10: Classic vs. trigonometric Bézier Curves.

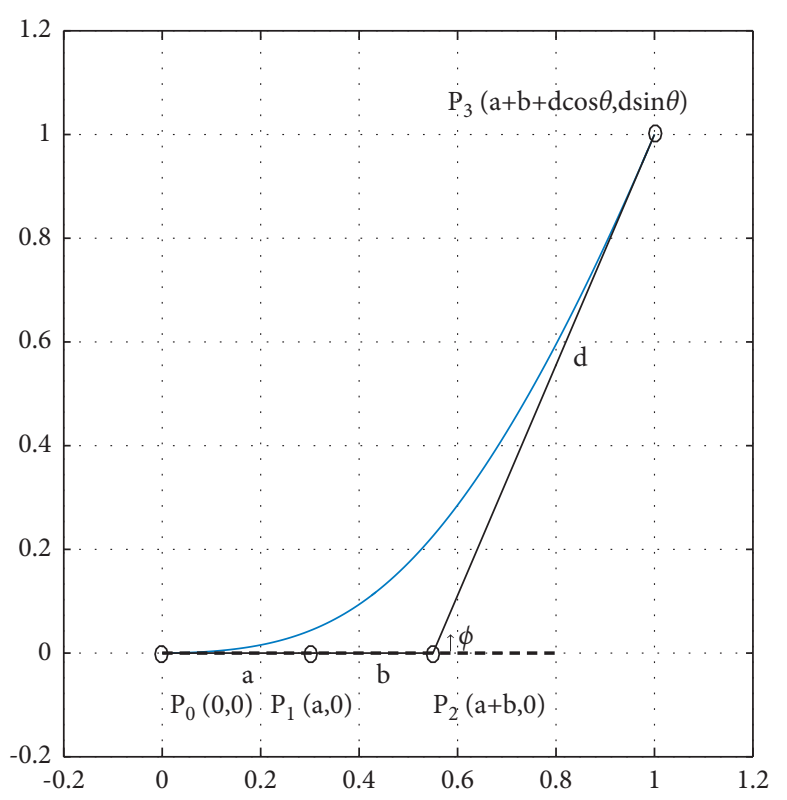

Figure 11: Planer CT-Bézier spiral curve and its control points. 
where $P^{\prime}=(1 / 2)\left(P_{1}+P_{2}\right)$.

$$
B(u)=(1-u)^{3} P_{0}+3(1-u)^{2} t P_{1}+3(1-u) u^{2} P_{2}+u^{3} P_{3},
$$

Proof. After some computation, $r(0)=P_{0}=B(0)$ and $r(1)=P_{3}=B(1)$.

Since

$$
\begin{aligned}
B\left(\frac{1}{2}\right)-P^{\prime} & =\frac{1}{8}\left(P_{0}-P_{1}-P_{2}+P_{3}\right) \\
r\left(\frac{1}{2}\right)-P^{\prime} & =\frac{1}{4}\left((\sqrt{ } 2-1)(\sqrt{ } 2-1+m)\left(P_{0}+P_{3}\right)-(\sqrt{ } 2-1)(\sqrt{ } 2-1+m)\left(P_{1}+P_{3}\right)\right. \\
& =\frac{1}{2}(\sqrt{ } 2-1)(\sqrt{ } 2-1+m)\left(P_{0}-P_{1}-P_{2}+P_{3}\right) \\
& =4(\sqrt{ } 2-1)(\sqrt{ } 2-1+m)\left(B\left(\frac{1}{2}\right)-P^{\prime}\right)
\end{aligned}
$$

Hence, it is proved.

Corollary 1. The CT-Bézier curve approaches to control polygon more than the Bézier curve for

$$
0 \leq m \leq \frac{(5-3 \sqrt{ } 2)}{4} .
$$

Corollary 2. CT-Bézier curve will be closer to classical Bézier curve when $m=(5-3 \sqrt{ } 2) / 4$, i.e., $r(1 / 2)=B(1 / 2)$.

The relation between the classic and trigonometric Bézier curve is given in Figure 10.

\section{Curvature and Spiral Curve}

A planer curve is defined by the set of points $r(u)=(Y(u), X(u))$ for real $u$. The tangent vector of $r(u)$ is given by $r^{\prime}(u)=\left(Y^{\prime}(u), X^{\prime}(u)\right)$. If $r^{\prime}(u) \neq 0=(0,0)$, then the signed curvature of $r(t)$ is defined as [11]

$$
k(t)=\frac{\overrightarrow{r^{\prime}(u)} \times \overrightarrow{r^{\prime \prime}(u)}}{\left\|\overrightarrow{r^{\prime}(u)}\right\|^{3}},
$$

where $\overrightarrow{r^{\prime}(u)} \times \overrightarrow{f^{\prime \prime}(u)}=r_{x}^{\prime} r_{y}^{\prime \prime}-r_{x}^{\prime \prime} r_{y}^{\prime}$.

Differentiate equation (19):

$$
k^{\prime}(t)=\frac{E(u)}{\left\|\overrightarrow{r^{\prime}(u)}\right\|^{5}},
$$

where $\left.E(u)=\left\{\overrightarrow{r^{\prime}(u)} \cdot \overrightarrow{r^{\prime \prime}(u)}\right\}\left\{\overrightarrow{r^{\prime}(u)} \times \overrightarrow{r^{\prime \prime}(u)}\right\}\right\}-3\left\{\overrightarrow{r^{\prime}(u)}\right.$ $\left.\times \overrightarrow{f^{\prime \prime}(u)}\right\} \overrightarrow{f^{\prime}(u)} \cdot \overrightarrow{f^{\prime \prime}(u)}$.

7.1. Planer Cubic Trigonometric Bézier Spiral Segment. Given a starting point $p_{0}$ at origin, i.e., $p_{0}=(0,0)$, the other points are

$$
\begin{aligned}
& p_{1}=p_{0}+a t_{0}, \\
& p_{2}=p_{1}+b t_{0}, \\
& p_{3}=p_{2}+d \cos \theta t_{0}+d \sin \theta n_{0},
\end{aligned}
$$

where $\left|p_{1}-p_{0}\right|=a,\left|p_{2}-p_{1}\right|=b$, and $\left|p_{3}-p_{2}\right|=d$. Here, $\theta$ is a positive angle from $\left|p_{2}-p_{1}\right|$ to $\left|p_{3}-p_{2}\right|$. The tangent unit vectors and the unit normal vector at the beginning points of the Bézier curve, see Figure 11, from [4] are

$$
\begin{aligned}
r(u)= & \left(1-\sin \frac{\pi}{2} u\right)\left[\left(1-\sin \frac{\pi}{2} u\right)+m \sin \frac{\pi}{2} u\right] p_{0}+\sin \frac{\pi}{2} u\left(1-\sin \frac{\pi}{2} u\right)(2-m) p_{1} \\
& +\cos \frac{\pi}{2} u\left(1-\cos \frac{\pi}{2} u\right)(2-m) p_{2}+\left(1-\cos \frac{\pi}{2} u\right)\left[\left(1-\cos \frac{\pi}{2} u\right)+m \cos \frac{\pi}{2} u\right] p_{3} .
\end{aligned}
$$


Now, equation (22) can be written as

$$
r(t)=(X(t), Y(t))
$$

$$
\begin{aligned}
X(u)= & \sin \frac{\pi}{2} u\left(1-\sin \frac{\pi}{2} u\right)(2-m) a+\cos \frac{\pi}{2} u\left(1-\cos \frac{\pi}{2} u\right)(2-m)(a+b) \\
& +\left(1-\cos \frac{\pi}{2} u\right)\left[\left(1-\cos \frac{\pi}{2} u\right)+m \cos \frac{\pi}{2} u\right](a+b+d \cos \theta), \\
Y(t)= & \left(1-\cos \frac{\pi}{2} u\right)\left[\left(1-\cos \frac{\pi}{2} u\right)+m \cos \frac{\pi}{2} u\right](d \sin \theta) .
\end{aligned}
$$

The more general form for spiral segment is now obtained by taking the derivatives of the curvature of (12). The first three derivatives of equations (23) and (24) are

$$
\begin{aligned}
X^{\prime}(u)= & \left(\frac{\pi}{2}\right)\left[(2-m) a\left[\cos \frac{\pi}{2} u-2 \cos \frac{\pi}{2} u \sin \frac{\pi}{2} u\right]+(2-m)(a+b)\left[2 \cos \frac{\pi}{2} u \sin \frac{\pi}{2} u-\sin \frac{\pi}{2} u\right]\right. \\
& \left.+\left(\sin \frac{\pi}{2} u\right)\left(2-m-2 \cos \frac{\pi}{2} u(1-m)\right)\right](a+b+d \cos \theta), \\
X^{\prime \prime}(u)= & \left(\frac{\pi}{2}\right)^{2}\left[(2-m)\left(\cos \frac{\pi}{2} u\left(2 b \cos \frac{\pi}{2} u-a-b\right)-\sin \frac{\pi}{2} u\left(a+2 b \sin \frac{\pi}{2} u\right)\right)\right. \\
& +\left[\left(\cos \frac{\pi}{2} u\right)\left(2-m-2 \cos \frac{\pi}{2} u(1-m)\right)+\left(\sin ^{2} \frac{\pi}{2} u\right)(2-2 m)\right](a+b+d \cos \theta), \\
X^{\prime \prime \prime}(u)= & \left(\frac{\pi}{2}\right)^{3}\left[(2-m)\left(\sin \frac{\pi}{2} u\left(a+b-8 b \cos \frac{\pi}{2} u\right)-a \cos \frac{\pi}{2} u\right)\right. \\
& +\left[5 \sin \frac{\pi}{2} u \cos \frac{\pi}{2} u(1-m)+\left(\sin \frac{\pi}{2} u+\cos \frac{\pi}{2} u\right)(m-1)+1\right](a+b+d \cos \theta), \\
Y^{\prime}(u)= & \left(\frac{\pi}{2}\right)\left(\sin \frac{\pi}{2} u\right)\left(2-m-2 \cos \frac{\pi}{2} u(1-m)\right)(d \sin \theta), \\
Y^{\prime \prime}(u)= & \left.\left(\frac{\pi}{2}\right)^{2}\left[\cos \frac{\pi}{2} u\right)\left(2-m-2 \cos \frac{\pi}{2} u(1-m)\right)+\left(\sin \frac{2}{2} u\right)(2-2 m)\right](d \sin \theta), \\
Y^{\prime \prime \prime}(u)= & \left(\frac{\pi}{2}\right)^{3}\left[5 \sin \frac{\pi}{2} u \cos \frac{\pi}{2} u(1-m)+\left(\sin \frac{\pi}{2} u+\cos \frac{\pi}{2} u\right)(m-1)+1\right](d \sin \theta) .
\end{aligned}
$$

It follows from equations (19), (25), (26), (28), and (29) that the curvature of equation (23) at $u=0$ and $u=1$ are, respectively,

$$
\begin{aligned}
k(0) & =\frac{m d \sin \theta}{((2-m) a)^{2}}, \\
k(1) & =\frac{\sin \theta[(2-2 m) d \cos \theta-((2-2 m)(a+b+d \cos \theta-(2-m)(a+2 b)))]}{((2-m) d)^{2}},
\end{aligned}
$$


and by using equation (20), we obtain

$$
\begin{aligned}
k^{\prime}(0) & =\frac{-3 m d^{2} \sin ^{2} \theta}{((2-m) a)^{3}}, \\
k(1) & =\frac{-3 \sin ^{2} \theta\left[((2-2 m) d \cos \theta)^{2}-((2-2 m)(a+b+d \cos \theta-(2-m)(a+2 b)))^{2}\right]}{((2-m) d)^{3}} .
\end{aligned}
$$

From equations (32) and (33), we conclude that the curve given in equation (22) is not a Bloss curve because it does not satisfy the conditions. However, if we put $\theta=0$, then curvature and its derivative values drop to zero; $r(u)$ is not a curve, it is a line. Thus, a cubic trigonometric Bézier Bloss curve is nothing but just a straight line.

\section{Conclusions}

New trigonometric cubic Bézier-like functions have been proposed in this work. Proposed bases' functions and curves satisfy the basic properties and have been proved. Open and closed curves with different control points and shape parameters have been constructed using proposed basis to check its applicability and flexibility. Furthermore, cubic trigonometric Bézier curve behave like a classical Bézier curve and have been proved. In the end, trigonometric spiral curve segment has also been constructed using cubic trigonometric functions, which indicate that proposed basis functions can be used in CAD/CAM modelling especially road and railway track designing.

8.1. Limitations and Future Work. The proposed bases' functions work well for all type of curves such as open and closed and are a good addition in literature; however, functions can be improved by increasing the interval of the free-shape parameter. In our future work, we will use the proposed work for the construction of craniofacial fracture and will develop the surface.

\section{Data Availability}

No data were used to support this study.

\section{Conflicts of Interest}

The authors declare that they have no conflicts of interest or personal relationships that could have appeared to influence the work reported in this paper.

\section{Authors' Contributions}

All authors equally contributed to this work. All authors read and approved the final manuscript.

\section{References}

[1] A. Majeed, A. R. Mt Piah, and Z. Ridzuan Yahya, "Surface reconstruction from parallel curves with application to parietal bone fracture reconstruction," PLoS one, vol. 11, no. 3, Article ID e0149921, 2016.

[2] A. Majeed, A. R. Mt Piah, R. U. Gobithaasan, and Z. R. Yahya, "Craniofacial reconstruction using rational cubic ball curves," PloS one, vol. 10, no. 4, Article ID e0122854, 2015.

[3] I. Schoenberg, "On trigonometric spline interpolation," Indiana University Mathematics Journal, vol. 13, no. 5, pp. 795-825, 1964.

[4] T. Lyche and R. Winther, "A stable recurrence relation for trigonometric B-splines," Journal of Approximation Theory, vol. 25, no. 3, pp. 266-279, 1979.

[5] R. Sharma, "Cubic trigonometric bzier curve with shape parameter," International Journal of Innovative Research in Computer and Communication Engineering, vol. 4, no. 4, pp. 7718-7723, 2016.

[6] X.-A. Han, Y. Ma, and X. Huang, "The cubic trigonometric Bézier curve with two shape parameters," Applied Mathematics Letters, vol. 22, no. 2, pp. 226-231, 2009.

[7] D. J. Walton and D. S. Meek, "A further generalisation of the planar cubic Bézier spiral," Journal of Computational and Applied Mathematics, vol. 236, no. 11, pp. 2869-2882, 2012.

[8] A. Ayar and B. Sahin, "Trigonometric Bézier-like curves and transition curves," Applicationes Mathematicae, pp. 1-29, 2021.

[9] Y. Zhu and X. Han, "Curves and surfaces construction based on new basis with exponential functions," Acta applicandae mathematicae, vol. 129, no. 1, pp. 183-203, 2014.

[10] B. Uzma, M. Abbas, M. N. H. Awang, and J. M. Ali, "The quadratic trigonometric Bzier curve with single shape parameter," Journal of basic and applied scientific research, vol. 2, no. 3, pp. 2541-2546, 2012.

[11] S. Maqsood, M. Abbas, G. Hu, A. L. A. Ramli, and K. T. Miura, "A novel generalization of trigonometric bézier curve and surface with shape parameters and its applications," Mathematical Problems in Engineering, vol. 2020, no. 1, 25 pages, Article ID 4036434, 2020.

[12] T. Bosner and M. Rogina, "Numerically stable algorithm for cycloidal splines," Annali dell'Universita di Ferrara, vol. 53, no. 2, pp. 189-197, 2007.

[13] T. Wen, G.-Z. Wang, Trigonometric polynomial uniform B-spline with shape parameter," Chinese Journal of computers. Chinese edition, vol. 28, no. 7, p. 1192, 2005.

[14] L. Y. Lan, "Cubic Trigonometric non uniform spline curves and surfaces," Mathematical Problems in Engineering, vol. 2016, Article ID 7067408, 9 pages, 2016. 
[15] Y. Z. A. X. Han, "New Trigonometric basis possessing exponential shape parameters," Journal of Computational Mathematics, vol. 33, no. 6, pp. 642-684, 2015.

[16] U. Mishra, "A $\lambda \mu-B$ - spline curve with shape parameter," Journal of Innovative research in computer and communication engineering, no. 8, pp. 2320-9801, 2016.

[17] N. Chouby and A. Ojha, "Trigonometric splines with variable shape parameter," Journal of Mathematics, vol. 38, 2008.

[18] K. Wang and G. Zhang, "New trigonometric basis possessing denominator shape parameter," Mathematics problems in Engineering, vol. 2018, Article ID 9569834, 25 pages, 2018.

[19] E. Troll, "Constrained modification of the Cubic trigonometric Bézier curve with two shape parameters," Annels Mathematics et Inforrmaticae, vol. 43, pp. 145-156, 2014.

[20] X. Han, "Cubic Trigonometric polynomial curves with a shape parameter," Computer Aided Geometric Design, vol. 21, no. 6, pp. 535-548, 2004.

[21] M. Dube and R. Sharma, "Quadratic NUAT-B-spline curves with multiple shape parameters," International Journal of Machine Intelligence, vol. 3, no. 1, pp. 18-24, 2011.

[22] X. Han, "Quadratic trigonometric polynomial curves with a shape parameter," Computer Aided Geometric Design, vol. 19, no. 7, pp. 503-512, 2002.

[23] X. Han, "Piecewise quadratic trigonometric polynomial curves," Mathematics of Computation, vol. 72, no. 243, pp. 1369-1378, 2003.

[24] W. Xie and J. Li, "C3 cubic trigonometric B-spline curves with a real parameter," Journal of the National Science Foundation of Sri Lanka, vol. 46, no. 1, 2018.

[25] H. Hang, X. Yao, Q. Li, and M. Artiles, "Cubic B-Spline curves with shape parameter and their applications," Mathematical Problems in Engineering, vol. 2017, Article ID 3962617, 7 pages, 2017.

[26] G. Hu, J. Wu, and X. Qin, "A new approach in designing of local controlled developable H-Bézier surfaces," Advances in Engineering Software, vol. 121, pp. 26-38, 2018.

[27] I. Kovcs and T. Vrady, "P-Bzier and P-Bspline curvesnew representations with proximity control," Computer Aided Geometric Design, vol. 62, pp. 117-132, 2018.

[28] M. Shafiq, M. Abbas, K. M. Abualnaja, M. J. Huntul, A. Majeed, and T. Nazir, "An efficient technique based on cubic B-spline functions for solving time-fractional advection diffusion equation involving Atangana-Baleanu derivative," Engineering with Computers, pp. 1-17, 2021.

[29] M. J. Huntul, M. Tamsir, A. A. H. Ahmadini, and S. R. Thottoli, "A novel collocation technique for parabolic partial differential equations," Ain Shams Engineering Journal, 2021. 